\title{
Diagnostic relevance of neutrophil gelatinase- associated lipocalin in early detection of acute kidney injury
}

\author{
Madhusudhan Mahadevaiah ${ }^{1,2}$, Murali Mohan Nidasale Thimmaiah ${ }^{2}$, Venu Sashank Yerramsetty ${ }^{2 *}$, Jeevan \\ Kumar'2, Ranjith Kumar ${ }^{2}$
}

'Emergency Physician, General Hospital, Nanjungud, Mysore, India 2Department of Emergency Medicine, Vydehi Hospital College: Vydehi Institute of Medical Sciences, ajiv Gandhi University of Health Sciences (RGUHS), Bangalore, India

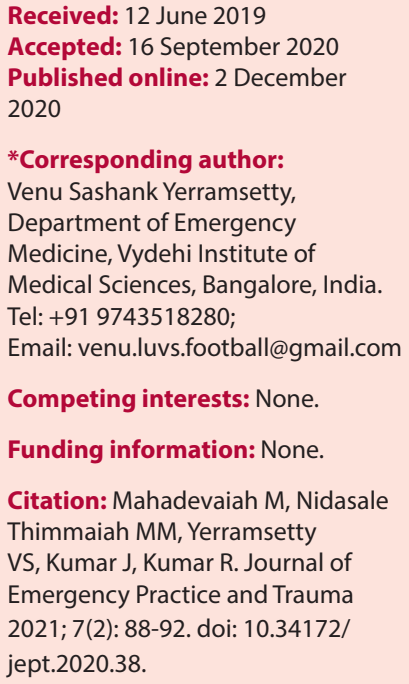

\begin{abstract}
Objective: To evaluate the predictive and diagnostic accuracy of neutrophil gelatinaseassociated lipocalin (NGAL) in acute kidney injury (AKI) and also to predict the renal replacement therapy (RRT) using NGAL as a marker.

Methods: This prospective study was conducted among the patients admitted to intensive care units. Plasma samples were collected 24 hours after admission and NGAL was measured using Triage ${ }^{\oplus}$ NGAL test, a specific point of care test which is based on the mechanism of fluorescence immunoassay. The diagnostic accuracy of plasma NGAL (pNGAL) to predict AKI in critically ill patients of ICU was assessed by applying receiver operator curve (ROC) analysis and calculating the area under the curve (AUC).

Results: In this study, 100 patients with the mean age of $49.56 \pm 19.2$ years were included for the period of 18 months. The blood samples were withdrawn from the patients 24 and 44 hours after admission. Totally, $55 \%(n=55)$ of ICU patients were diagnosed with AKI. Plasma NGAL level was significantly increased in AKI patients as compared to non-AKI patients $(742.65 \pm 734.72$ vs. $255.62 \pm 440.09 \mu \mathrm{g} / \mathrm{L} ; P<0.01)$. The sensitivity and specificity of NGAL for diagnosing AKI was $83.6 \%$ and $88.9 \%$, respectively. The overall diagnostic accuracy was $86 \%$. Diagnostic accuracy of NGAL for requirement of RRT was $51 \%$.

Conclusion: Plasma NGAL is a reliable marker for patients with AKI in ICU, in case the cause of kidney injury is not known. In addition, NGAL also predicts the RRT need based on AKI severity.

Keywords: Neutrophil gelatinase-associated Lipocalin, Acute kidney injury, Renal replacement therapy
\end{abstract}

\section{Introduction}

Acute kidney injury (AKI) is an alarming pathological event resulting in rapid decrease in the kidney function and can lead to kidney cell damage. The hallmark feature of AKI is the decrease in glomerular filtration rate $(1,2)$. Moreover, during AKI there is a substantial elevation of kidney diagnostic indices such as serum creatinine ( $\mathrm{sCr}$ ), and blood urea nitrogen (BUN) with a corresponding decrease in urine output (3). The patients affected with AKI have significant burden with increased mortality rate, hospital stay, intensive care unit admissions and hospital cost (4). For cases in which there is no timely diagnosis and accurate management, the resultant would be poor clinical outcome (5). Up to the present there is no standard management protocol for AKI, albeit renal replacement therapy (RRT) has displayed a significant outcome in the reduction of AKI and the success rate is associated with the various factors such as time of therapy initiation and completion $(6,7)$. The present definition of AKI is solely based on loss of kidney function without considering the ultra- structural changes in the kidney. Meanwhile, clinically $\mathrm{sCr}$ is employed as a reliable marker to assess the kidney function but its accuracy is very limited during acute changes. Further, sCr level is largely dependent upon various factors such as age, sex, BMI, food intake, drugs, and hydration status (8).

Neutrophil gelatinase-associated lipocalin (NGAL) is a glycoprotein of low molecular weight which belongs to the family of lipocalin. It has the capacity to bind with low size lipophilic components derived from bacterial lipopolysaccharide (9). Generally, human NGAL is located in neutrophils in the form of secondary granules (10). In 
normal physiological conditions, in organs like kidney, small intestine, colon and lung, NGAL is expressed at low concentrations (11). However, during disease conditions like epithelial cell injury the protein level of NGAL is upregulated in various organs like lungs, colon and kidney (12) as well as in tumor of breast cancer, colon and pancreas $(13,14)$. Generally, in blood and urine, NGAL exists in 3 forms such as monomer, homodimer and heterodimer which is connected to gelatinase through linkage (15). NGAL is used as a reliable AKI biomarker since it is the chief protein expressed rapidly during ischemic condition or as a result of various stress factors in renal tissues. Furthermore, during renal injury there is a substantial loss of renal cell membrane integrity and due to this the NGAL is rapidly released into the bloodstream and urine (16). Previous studies show the diagnostic accuracy of NGAL as well as a prominent marker in the early diagnosis of AKI (17). In addition, it has been employed as an effective predictor for the requirement of RRT and dialysis in AKI patients $(18,19)$. In this backdrop, the current study was conducted to evaluate the predictive and diagnostic accuracy of NGAL and also the prediction of RRT based on NGAL values in AKI patients.

\section{Methods}

This was a prospective study conducted for a period of 18 months and included all the Emergency Department admissions at Vydehi Institute of Medical Sciences and Research Centre, Bangalore which fitted the inclusion/ exclusion criteria. The patients were followed up for the period of 4 weeks. Informed written consent was granted from all patients included in the study.

The inclusion criteria encompassed patients with varying ages presenting to the Emergency Department having a complaint of decreased urinary output and features of AKI.

Patients affected with myeloproliferative disorder, peritonitis, acute chronic obstructive pulmonary disease exacerbation and urinary tract infection were excluded from the study.

AKI was defined based on the criteria of creatinine and urine output stated in RIFLE (Risk-Injury-Failure-LossEnd stage renal disease) classification (20). The kidney function at baseline was defined as least value of $\mathrm{sCr}$ measured during the past 3 months. In patients where the previous sCr was not measured it was calculated by solving the MDRD equation $(20,21)$. Initial RIFLE denotes the RIFLE class on day 1 of AKI. RIFLE-max is the alarming RIFLE class exhibited by the patient during the ICU admission. In patients with AKI, the kidney functions during discharge from ICU were as follows: Complete renal function recovery when $\mathrm{sCr}<150 \%$ as that of the baseline; partial renal function recovery when $\mathrm{sCr}<150-199 \%$ as that of the baseline; no renal function recovery when $\mathrm{sCr} \geq 200 \%$ as that of baseline or the patient was on RRT.
The blood collection for analysis was done daily and processed for NGAL estimation within 1 hour. The collected samples were subjected to centrifugation at 3500RPM for ten minutes and the supernatant was stored at $-80^{\circ} \mathrm{C}$ before measurement. Plasma NGAL (pNGAL) was estimated using the Triage ${ }^{\circledR}$ NGAL Test (Biosite Inc, San Diego, CA), a point-of-care fluorescence-based immunoassay.

Data analysis was done by using SPSS software version 21 with receiver operator curve (ROC). All the demographic variables were represented using percentage, and mean \pm standard deviation. Sensitivity, specificity, positive predictive value (PPV), negative predictive value (NPV), true positives and true negatives of NGAL test were found by using sCr test as gold standard method. Gold Standard for $\mathrm{AKI}$ is the value of $\mathrm{sCr}$ calculated by approved Laboratory investigation and AKI graded according to RIFLE criteria. Prediction of AKI and calculation of baseline SCr using Risk-Injury-Failure-Loss-End Stage (RIFLE-sCr) was proposed by the Acute Dialysis Quality Initiative (ADQI) group. A twofold increase in $\mathrm{sCr}$ from baseline value is considered as AKI. Cut off $150 \mathrm{ng} / \mathrm{mL}$ of Plasma NGAL was used for the prediction of AKI.

\section{Results}

The current study was done on 100 patients admitted to ICU. The mean age of patients $(n=100)$ was $49.56 \pm 19.2$ years with a male preponderance of $58 \%$. Majority of the subjects displayed the Glasgow coma scale score between 12 and 15 (93\%). The major complication during ICU admission was mainly due to cardiac failure (33\%) followed by sepsis (26\%) and acute gastroenteritis (21\%). Table 1 shows the general and clinical characteristics of the patients.

In this study, out of 100 patients admitted to ICU, 55

Table 1. Demographics and clinical details of patients $(n=100)$ admitted to ICU

\begin{tabular}{lc}
\hline Characteristics & No. $(\%)$ \\
\hline Age & $49.56 \pm 19.2$ years \\
Sex & $58(58)$ \\
$\quad$ Male & $42(42)$ \\
$\quad$ Females & \\
GCS Score & $2(2)$ \\
$\leq 8$ & $5(5)$ \\
9 to 12 & $93(93)$ \\
12 to 15 & \\
\hline Final diagnosis during ICU admission & $33(33)$ \\
\hline Cardiac failure & $26(26)$ \\
\hline Sepsis & $21(21)$ \\
\hline Acute GE & $7(7)$ \\
\hline Acute coronary syndrome & $5(5)$ \\
\hline Shock & $2(2)$ \\
\hline Seizures & $2(2)$ \\
\hline Snake bite & $2(2)$ \\
\hline Toxin ingestion & $1(1)$ \\
\hline Diabetic ketoacidosis & \\
\hline GE, gastro enteritis &
\end{tabular}


patients (55\%) developed AKI and the prevalence was found to be $55 \%$. Further, no significant difference $(P>0.05)$ was observed in patients with and without AKI with respect to Glasgow Coma Scale, respiratory rate, partial pressure of oxygen and heart rate. The blood pressure was found to be normal in majority of the patients with AKI and non-AKI and it was found to be insignificant. Moreover, there was no significant alteration in complete blood count and electrolytes levels between AKI and non-AKI patients. However, blood gas analysis revealed that the metabolic acidosis was observed in $67.3 \%$ of cases with AKI as compared to $46.7 \%$ of nonAKI cases and it was insignificant $(P=0.14)$.

In the present study, there was a significant $(P<0.01)$ decrease in urine output in AKI subjects at 6 hours, 12 hours and 24 hours as compared to non-AKI patients (Table 2). Also, the blood urea and sCr at 2 hours and 24 hours significantly $(P<0.01)$ increased in AKI patients in comparison to non-AKI patients (Table 3 ).

Our study shows that the plasma NGAL level was significantly elevated in AKI subjects as compared to non-AKI subjects $(742.65 \pm 734.72$ vs $255.62 \pm 440.09$ $\mu \mathrm{g} / \mathrm{L} ; \quad P<0.01)$. Besides, the area under NGAL curve for the prediction of AKI development was found to be 0.846 with $95 \%$ CI of 0.76 to $0.93(P<0.01$; Figure 1$)$. The sensitivity, specificity, PPV and negative predictive of NGAL for diagnosing AKI was $83.6 \%, 88.9 \%$, 90.2\% and $81.6 \%$, respectively. By the same token, the overall diagnostic accuracy of NGAL in predicting AKI was found to be $86 \%$.

In this study, out of 100 ICU cases, 8 patients required RRT and in these patients the mean NGAL level was found to be $446.88 \pm 407.02 \mu \mathrm{g} / \mathrm{L}$ and the remaining 98 patients did not require RRT during their hospital admission and the NGAL level was found to be $530.15 \pm 682.05$ $\mu \mathrm{g} / \mathrm{L}$. However, the value was not statistically significant $(P=0.73)$. Also, the area under NGAL curve for the prediction of RRT requirement was found to be 0.499 with $95 \%$ CI of 0.29 to 0.707 ( $P$ 0.99; Figure 2). We assume

Table 2. Urine output among AKI and non-AKI subjects

\begin{tabular}{lccc}
\hline Urine output & AKI $(\mathbf{n}=\mathbf{5 5})(\mathbf{m L})$ & Non-AKI $(\mathbf{n}=\mathbf{4 5})(\mathbf{m L})$ & P value \\
\hline $6 \mathrm{~h}$ & $190.09 \pm 169.73$ & $346.33 \pm 214.60$ & $<0.01$ \\
$12 \mathrm{~h}$ & $378.61 \pm 301.89$ & $802.44 \pm 488.26$ & $<0.01$ \\
$24 \mathrm{~h}$ & $725.85 \pm 445.41$ & $1486.44 \pm 896.22$ & $<0.01$ \\
\hline
\end{tabular}

The values are represented as mean \pm SD. Comparison were done between AKI vs Non-AKI. $P$ value $<0.01$ was found to be statistically significant.

Table 3. Renal function test among AKI and non-AKI subjects

\begin{tabular}{lcccc}
\hline Renal function test & & AKI $(\mathbf{n = 5 5})$ & Non-AKI $(\mathbf{n = 4 5})$ & P value \\
\hline Blood Urea $(\mathrm{mg} / \mathrm{dL})$ & $2 \mathrm{~h}$ & $67.13 \pm 35.06$ & $40.09 \pm 23.44$ & $<0.01$ \\
& $24 \mathrm{~h}$ & $109.47 \pm 44.38$ & $42.58 \pm 18.62$ & $<0.01$ \\
Serum Creatinine & $2 \mathrm{~h}$ & $2.38 \pm 2.10$ & $1.25 \pm 0.88$ & $<0.01$ \\
(mg/dL) & $24 \mathrm{~h}$ & $2.94 \pm 1.42$ & $1.20 \pm 0.66$ & $<0.01$ \\
\hline
\end{tabular}

The values are represented as mean $\pm \mathrm{SD}$. Comparison were done between AKI vs Non-AKI. p value $<0.01$ was found to be statistically significant

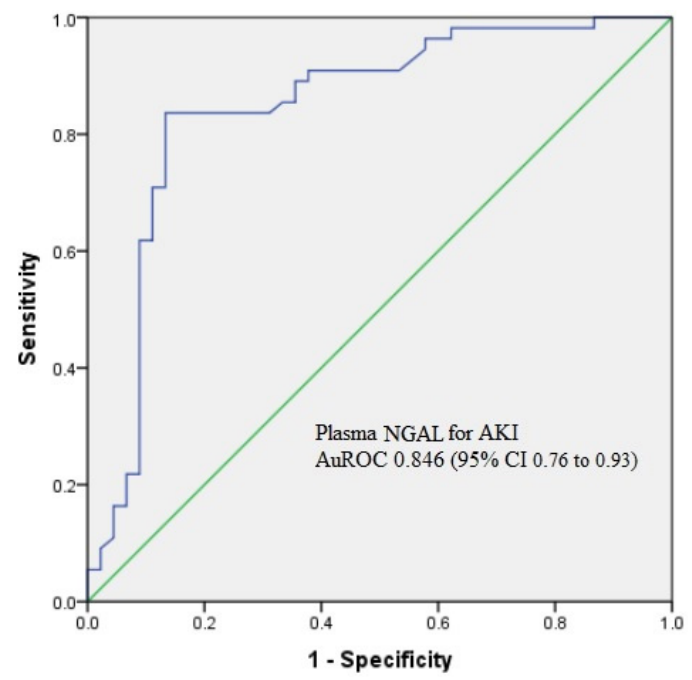

Figure 1. Receiver operator characteristic (ROC) curve for plasma NGAL. The area under the ROC is 0.846 (95\% Cl 0.76 to 0.93$)$, which displays a good performance for the prediction of AKI.

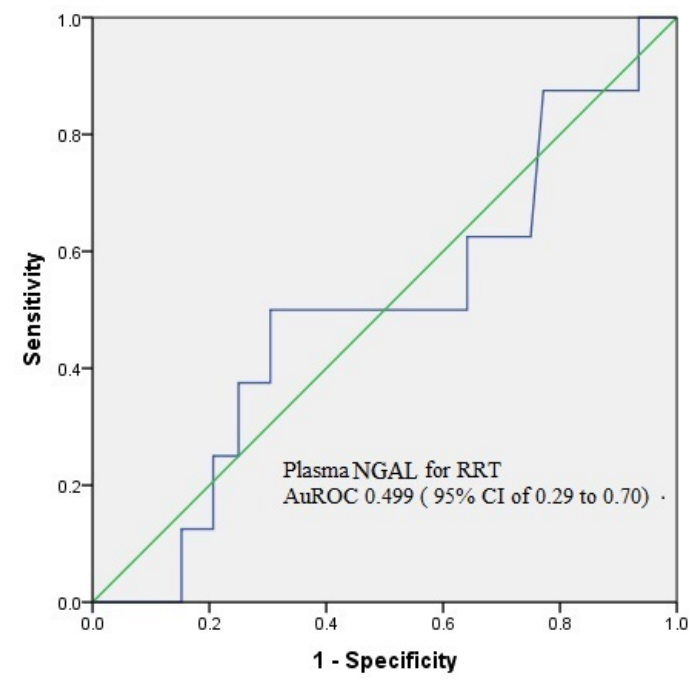

Figure 2. Receiver operator characteristic (ROC) curve for plasma NGAL for the requirement of RRT. The area under the ROC is 0.499 with $95 \% \mathrm{Cl}$ of 0.29 to 0.707 .

that NGAL is effective in predicting AKI other than RRT. The sensitivity, specificity, PPV and negative predictive of NGAL for analyzing the RRT requirement in AKI was found to be $62.5 \%, 50 \%, 9.2 \%$ and $93.9 \%$, respectively. The overall diagnostic accuracy of NGAL in predicting the requirement of RRT in AKI patients was found to be $51 \%$ (Table 4).

\section{Discussion}

In this study a prospective analysis was conducted to evaluate the diagnostic accuracy of plasma NGAL (pNGAL) for the rapid detection of AKI in ICU admitted patients. In our study, out of 100 patients admitted to ICU, 55 patients were diagnosed with AKI and the prevalence rate was $55 \%$. We observed that pNGAL level significantly increased in AKI patients as compared to the non-AKI 
Table 4. Diagnostic accuracy of NGAL for prediction of RRT requirement

\begin{tabular}{lc}
\hline NGAL for the prediction of RRT & Percent \\
\hline Sensitivity & 62.5 \\
Specificity & 50.00 \\
PPV & 9.8 \\
NPV & 93.9 \\
Accuracy & 51.0 \\
\hline
\end{tabular}

NGAL: neutrophil gelatinase-associated lipocalin; RRT: renal replacement therapy; NPV: negative predictive value; PPV: positive predictive value.

patients $(742.65 \mu \mathrm{g} / \mathrm{L}$ vs $255.62 \mu \mathrm{g} / \mathrm{L} ; P<0.01)$. Similar to our study, the research undertaken by Soto et al., showed an increase in the level of pNGAL in patients with AKI (146-174 ng/mL) estimated at various time ranges (22). Another study done by Khan et al. also displayed an elevated pNGAL level in AKI patients with a median range of 126$274 \mathrm{ng} / \mathrm{mL}$ (23). In the present study, area under the curve (AUC) of NGAL for the prediction of AKI development was 0.846 with $95 \% \mathrm{CI}$ of 0.763 to $0.93(P<0.01)$. In line with our study, researches done by Yamashita et al (24) and Dai et al (25) revealed an AUC of 0.94 (95\% CI, 0.88-0.97) and 0.92 (95\% CI, 0.84-0.96) for the accuracy of pNGAL as a predictor of AKI in ICU patients. In another study conducted by Md Ralib et al, a similar AUC was reported (95\% CI 0.74 to 0.87 ) for pNAGL in AKI prediction (26). In our study, the sensitivity, specificity, PPV and NPV of NGAL for diagnosing AKI was found to be $83.6 \%, 88.9 \%$, $90.2 \%$ and $81.6 \%$, respectively. However, in a study done by Hjortrup et al the sensitivity, specificity, PPV and NPV was found to be $58 \%, 76 \%, 45 \%$ and $85 \%$, respectively (27). We reported an overall diagnostic accuracy of $86 \%$ in predicting AKI. In a study conducted by Tecson et al, they reported a diagnostic accuracy of 75.45 which is less when compared to our study (28).

Mean of NGAL levels among cases with and without requirement of RRT was $446.88 \mu \mathrm{g} / \mathrm{L}$ and $530.15 \mu \mathrm{g} / \mathrm{L}$, respectively $(P=0.73)$. The area under NGAL curve for predicting RRT requirement was 0.499 with $95 \%$ CI of 0.29 to $0.707(P=0.99)$. The sensitivity and specificity of NGAL for predicting the requirement of RRT was $62.5 \%$ and $50 \%$ respectively with the overall diagnostic accuracy of $51 \%$. However, in our study NGAL was not observed to be a significant biomarker for the requirement of RRT which might be due to the relatively small sample of population $(n=8)$ requiring RRT to come to a valid statistical conclusion. The recent meta-analysis conducted on biomarkers for the prediction of RRT need shows that albeit with high heterogeneity, multiple studies show good predictive performance for NGAL, however, the authors are unable to substantiate the efficacy of NGAL compared to other biomarkers in RRT prediction (29).

\section{Conclusion}

The present study reveals that plasma NGAL is a very effective marker in rapid diagnosis of AKI in ICU admitted patients. Further, it enhances AKI diagnosis within 48 hours before routine clinical diagnosis as per RIFLE criteria. It is also effective in predicting RRT need and effectively correlates with AKI severity as well as overall illness. Future studies are highly recommended to validate the accuracy of urine and plasma NGAL by measuring its sensitivity and specificity from different clinical samples among a large set of ICU admitted patients with different clinical conditions.

\section{Authors' contribution}

MM, NTM framed the study protocol. MM, RK, YVS conducted the study. YVS, JK performed data analysis and manuscript drafting.

\section{Ethical issue}

The study was approved by Vydehi Institute of Medical Sciences and Research Centre, Bangalore with ethical clearance number, ECR/747/Inst/KA/2015.

\section{References}

1. Molitoris BA. Measuring glomerular filtration rate in acute kidney injury: yes, but not yet. Crit Care 2012; 16(5): 158. doi: 10.1186/cc11482.

2. Shapiro NI, Trzeciak S, Hollander JE, et al. The diagnostic accuracy of plasma neutrophil gelatinase-associated lipocalin in the prediction of acute kidney injury in emergency department patients with suspected sepsis. Ann Emerg Med. 2010;56(1):52-59.e1. doi:10.1016/j. annemergmed.2010.02.010.

3. Susantitaphong P, Cruz DN, Cerda J, Abulfaraj M, Alqahtani F, Koulouridis I, et al. World incidence of AKI: a meta-analysis. Clin J Am Soc Nephrol 2013; 8(9): 1482-93. doi: $10.2215 /$ cjn.00710113.

4. Hobson C, Ozrazgat-Baslanti T, Kuxhausen A, Thottakkara P, Efron PA, Moore FA, et al. Cost and mortality associated with postoperative acute kidney injury. Ann Surg 2015; 261(6): 1207-14. doi: 10.1097/sla.0000000000000732.

5. Kellum JA, Chawla LS, Keener C, Singbartl K, Palevsky PM, Pike FL, et al. The effects of alternative resuscitation strategies on acute kidney injury in patients with septic shock. Am J Respir Crit Care Med 2016; 193(3): 281-7. doi: 10.1164/rccm.201505-0995OC.

6. Ronco C, Ricci Z, De Backer D, Kellum JA, Taccone FS, Joannidis $\mathrm{M}$, et al. Renal replacement therapy in acute kidney injury: controversy and consensus. Crit Care 2015; 19(1): 146. doi: 10.1186/s13054-015-0850-8.

7. Bhatt GC, Das RR. Early versus late initiation of renal replacement therapy in patients with acute kidney injury-a systematic review \& meta-analysis of randomized controlled trials. BMC Nephrol 2017; 18(1): 78. doi: 10.1186/s12882017-0486-9.

8. Sharfuddin AA, Molitoris BA. Pathophysiology of ischemic acute kidney injury. Nat Rev Nephrol 2011; 7(4): 189-200. doi: 10.1038/nrneph.2011.16.

9. Cai L, Rubin J, Han W, Venge P, Xu S. The origin of multiple molecular forms in urine of HNL/NGAL. Clin J Am Soc Nephrol 2010; 5(12): 2229-35. doi: 10.2215/cjn.00980110.

10. Borregaard N, Cowland JB. Neutrophil gelatinaseassociated lipocalin, a siderophore-binding eukaryotic 
protein. Biometals 2006; 19(2): 211-5. doi: 10.1007/s10534005-3251-7.

11. Xu S, Venge P. Lipocalins as biochemical markers of disease. Biochim Biophys Acta 2000; 1482(1-2): 298-307. doi: 10.1016/s0167-4838(00)00163-1.

12. Mishra J, Mori K, Ma Q, Kelly C, Barasch J, Devarajan P. Neutrophil gelatinase-associated lipocalin: a novel early urinary biomarker for cisplatin nephrotoxicity. Am J Nephrol 2004; 24(3): 307-15. doi: 10.1159/000078452.

13. Wenners AS, Mehta K, Loibl S, Park H, Mueller B, Arnold $\mathrm{N}$, et al. Neutrophil gelatinase-associated lipocalin (NGAL) predicts response to neoadjuvant chemotherapy and clinical outcome in primary human breast cancer. PLoS One 2012; 7(10): e45826. doi: 10.1371/journal.pone.0045826.

14. McLean MH, Thomson AJ, Murray GI, Fyfe N, Hold GL, ElOmar EM. Expression of neutrophil gelatinase-associated lipocalin in colorectal neoplastic progression: a marker of malignant potential? Br J Cancer 2013; 108(12): 2537-41. doi: 10.1038/bjc.2013.264.

15. Mårtensson J, Bellomo R. The rise and fall of NGAL in acute kidney injury. Blood Purif 2014; 37(4): 304-10. doi: 10.1159/000364937.

16. Devarajan P. Emerging urinary biomarkers in the diagnosis of acute kidney injury. Expert Opin Med Diagn 2008; 2(4): 387-98. doi: 10.1517/17530059.2.4.387.

17. Antonucci E, Lippi G, Ticinesi A, Pigna F, Guida L, Morelli I, et al. Neutrophil gelatinase-associated lipocalin (NGAL): a promising biomarker for the early diagnosis of acute kidney injury (AKI). Acta Biomed 2014; 85(3): 289-94.

18. Cruz DN, de Cal M, Garzotto F, Perazella MA, Lentini P, Corradi V, et al. Plasma neutrophil gelatinase-associated lipocalin is an early biomarker for acute kidney injury in an adult ICU population. Intensive Care Med 2010; 36(3): 444-51. doi: 10.1007/s00134-009-1711-1.

19. Endre ZH, Pickering JW, Walker RJ, Devarajan P, Edelstein CL, Bonventre JV, et al. Improved performance of urinary biomarkers of acute kidney injury in the critically ill by stratification for injury duration and baseline renal function. Kidney Int 2011; 79(10): 1119-30. doi: 10.1038/ ki.2010.555.

20. Bellomo R, Ronco C, Kellum JA, Mehta RL, Palevsky P. Acute renal failure - definition, outcome measures, animal models, fluid therapy and information technology needs: the Second International Consensus Conference of the Acute Dialysis Quality Initiative (ADQI) Group. Crit Care 2004; 8(4): R204-12. doi: 10.1186/cc2872.
21. Levey AS, Stevens LA, Schmid CH, Zhang YL, Castro AF 3rd, Feldman HI, et al. A new equation to estimate glomerular filtration rate. Ann Intern Med 2009; 150(9): 604-12. doi: 10.7326/0003-4819-150-9-200905050-00006.

22. Soto K, Papoila AL, Coelho S, Bennett M, Ma Q, Rodrigues B, et al. Plasma NGAL for the diagnosis of AKI in patients admitted from the emergency department setting. Clin J Am Soc Nephrol 2013; 8(12): 2053-63. doi: 10.2215/ cjn.12181212.

23. 23.Khan BD, Munir M, Khan F, Naqvi SM. Comparison of urine with plasma neutrophil gelatinase- associated lipocalin in detecting acute kidney injury after cardiopulmonary bypass surgery. Pak Armed Forces Med J 2013; 63(2): 179-83.

24. Yamashita T, Doi K, Hamasaki Y, Matsubara T, Ishii T, Yahagi N, et al. Evaluation of urinary tissue inhibitor of metalloproteinase- 2 in acute kidney injury: a prospective observational study. Crit Care 2014; 18(6): 716. doi: 10.1186/s13054-014-0716-5.

25. Dai X, Zeng Z, Fu C, Zhang S, Cai Y, Chen Z. Diagnostic value of neutrophil gelatinase-associated lipocalin, cystatin $\mathrm{C}$, and soluble triggering receptor expressed on myeloid cells-1 in critically ill patients with sepsis-associated acute kidney injury. Crit Care 2015; 19(1): 223. doi: 10.1186/ s13054-015-0941-6.

26. Md Ralib A, Mat Nor MB, Pickering JW. Plasma Neutrophil Gelatinase-Associated Lipocalin diagnosed acute kidney injury in patients with systemic inflammatory disease and sepsis. Nephrology (Carlton) 2017; 22(5): 412-9. doi: 10.1111/nep.12796.

27. Hjortrup PB, Haase N, Treschow F, Møller MH, Perner A. Predictive value of NGAL for use of renal replacement therapy in patients with severe sepsis. Acta Anaesthesiol Scand 2015; 59(1): 25-34. doi: 10.1111/aas.12427.

28. Tecson KM, Erhardtsen E, Eriksen PM, Gaber AO, Germain M, Golestaneh L, et al. Optimal cut points of plasma and urine neutrophil gelatinase-associated lipocalin for the prediction of acute kidney injury among critically ill adults: retrospective determination and clinical validation of a prospective multicentre study. BMJ Open 2017; 7(7): e016028. doi: 10.1136/bmjopen-2017-016028.

29. Klein SJ, Brandtner AK, Lehner GF, Ulmer H, Bagshaw SM, Wiedermann CJ, et al. Biomarkers for prediction of renal replacement therapy in acute kidney injury: a systematic review and meta-analysis. Intensive Care Med 2018; 44(3): 323-36. doi: 10.1007/s00134-018-5126-8. 